Revista de Metalurgia 52(2)

Abril-Junio 2016, e066

ISSN-L: 0034-8570

doi: http://dx.doi.org/10.3989/revmetalm.066

\title{
Estudio del comportamiento termo-mecánico de un acero microaleado de medio carbono durante un proceso de conformado en caliente usando una red neuronal artificial
}

\author{
Ignacio Alcelay, Esteban Peña, Anas Al Omar ${ }^{\bowtie}$ \\ Universidad Politécnica de Cataluña, EPSEM, Departamento de Ingeniería Mecánica, \\ Av. Bases de Manresa 61 - 73, 08240 Manresa, Barcelona, España \\ Autor para la correspondencia: anas.al.omar@upc.edu
}

Enviado: 19 Noviembre 2015; Aceptado: 25 Enero 2016; Publicado on-Line: 21 Junio 2016

RESUMEN: El comportamiento termo-mecánico de un acero microaleado de medio carbono ha sido analizado mediante una Red Neuronal Artificial (RNA). Las curvas de fluencia para el entrenamiento de la RNA han sido obtenidas mediante ensayos de compresión en caliente que se efectuaron a temperaturas que oscilaron entre $1150^{\circ} \mathrm{C}$ y $900{ }^{\circ} \mathrm{C}$ a incrementos de $50{ }^{\circ} \mathrm{C}$, y en un intervalo de velocidades de deformación que varió entre

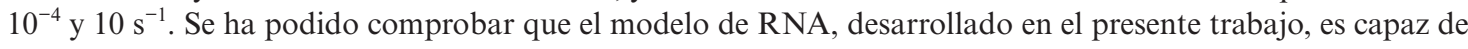
predecir con exactitud y eficiencia el comportamiento de fluencia en caliente del acero estudiado y existe un buen acuerdo entre los resultados experimentales y los resultados de la RNA. Para analizar la conformabilidad del acero microaleado se han construido mapas de procesado basados en el modelo dinámico de materiales (DMM) usando los valores de la tensión de fluencia obtenidos mediante la RNA. El estudio de los mapas revela los diferentes dominios de la fluencia plástica del acero estudiado y muestra la gran similitud entre los resultados experimentales y los resultados teóricos, por lo que el uso de la RNA puede constituir una alternativa muy interesante para el diseño y el estudio de los procesos de conformado en caliente.

PALABRAS CLAVE: Aceros microaleados; Conformado en caliente; Mapas de procesado; Modelo dinámico de materiales; Red neuronal artificial

Citation / Cómo citar este artículo: Alcelay, I., Peña, E., Al Omar, A. (2016) "Estudio del comportamiento termomecánico de un acero microaleado de medio carbono durante un proceso de conformado en caliente usando una red neuronal artificial”. Rev. Metal. 52(2):e066. doi: http://dx.doi.org/10.3989/revmetalm.066.

\begin{abstract}
Study of the thermo-mechanical behavior of medium carbon microalloyed steel during hot forming process using an artificial neural network. The thermo-mechanical behavior of medium carbon microalloyed steel has been analyzed by an Artificial Neural Network (ANN). The flow curves for training the ANN have been obtained from the hot compression tests, carried out over a temperature range varying from 900 to $1150{ }^{\circ} \mathrm{C}$ and at different true strain rates ranging from $10^{-4}$ to $10 \mathrm{~s}^{-1}$. It has been found that the ANN model developed in this study is capable to predict accurately and efficiently the flow behavior of the studied steel and there is a good agreement between the experimental results and the ANN results. To analyze the formability of the studied steel, processing maps have been constructed on the basis of the Dynamic Materials Model (DMM), using the ANN values of the flow stress. The study of maps reveals the different domains of the flow behavior of the studied steel and shows the great similarity between the experimental results and the theoretical results, so the use of the ANN can constitute an interesting alternative for design and study of hot forming processes.
\end{abstract}

KEYWORDS: Artificial neuronal network; Dynamic material model; Hot working; Microalloyed steels; Processing maps

Copyright: (C) 2016 CSIC. This is an open-access article distributed under the terms of the Creative Commons Attribution-Non Commercial (by-nc) Spain 3.0 License. 


\section{INTRODUCCIÓN}

La crisis económica y la necesidad de un desarrollo sostenible han obligado a la industria de automoción a utilizar nuevos aceros con el objetivo de reducir el peso de los automóviles (ahorro en combustible y menor emisión de $\mathrm{CO}_{2}$ ) y aumentar su resistencia al impacto (más seguridad en el vehículo y menos daños corporales). En este sentido, los aceros microaleados han sido ampliamente estudiados y utilizados en la industria, y su importancia estriba en el hecho de no necesitar tratamientos térmicos una vez conformada la pieza. Esto supone un ahorro económico muy interesante en costes de energía y tiempo (Naylor, 1998; Kaynar et al., 2013; Hui et al., 2015). Para poder optimizar las propiedades mecánicas de este tipo de aceros y fabricar piezas de alta precisión dimensional, los parámetros de control del proceso de conformado deben ser elegidos cuidadosamente. Durante un proceso de conformado en caliente, el comportamiento de fluencia del material está notablemente influenciado por factores mecánicos y microestructurales (Da Silva et al., 2006). La influencia de estos factores sobre la tensión de fluencia es muy complicada de describir mediante una ecuación constitutiva simple, por el gran número de fenómenos metalúrgicos interconectados que puede exhibir el material durante su transformación termo-mecánica: inestabilidad plástica, endurecimiento por deformación, restauración dinámica y recristalización dinámica, etc. Por ello, para controlar las propiedades mecánicas finales y obtener una mejor precisión dimensional en el producto conformado es imprescindible modelizar el comportamiento de fluencia del material para poder optimizar los parámetros de control del proceso de conformado (Jahazi y Eghbali, 2001; Ceschini et al., 2013). En el intento de proporcionar ecuaciones constitutivas apropiadas, varios modelos matemáticos han sido propuestos para caracterizar, a partir de datos experimentales, el comportamiento de los aceros microaleados en procesos de conformado en caliente (Cabrera et al., 1997; Shaban y Egbali, 2010; Akbari et al., 2015). La complejidad microestructural de este tipo de aceros y la no linealidad de su comportamiento de fluencia hacen difícil y complejo el desarrollo de una ecuación constitutiva capaz de predecir con exactitud el comportamiento del material. Los métodos convencionales usan métodos de regresión para ajustar modelos existentes a los datos experimentales y así obtener los parámetros de la ecuación constitutiva. Pero, cuando se añaden nuevos datos, la mayoría de los parámetros ya calculados cambian y habrá que volver a reajustar el modelo para obtener nuevos parámetros. En consecuencia, esta vía puede ser muy lenta y puede reducir considerablemente la exactitud de los resultados obtenidos. Por el contrario, las Redes Neuronales Artificiales (RNA) no requieren una forma específica de ecuaciones y no necesitan tener en cuenta los mecanismos de deformación, sólo requieren datos suficientes y significativos de entrada/salida. La capacidad de los modelos RNA para la descripción de los comportamientos no lineales y la interacción entre los diferentes mecanismos de deformación ha sido ampliamente demostrada en numerosas aplicaciones en ciencias de materiales (Chai et al., 2012; Gupta et al., 2012; Alcelay et al., 2014; Zhao et al., 2014; Reddy et al., 2015).

Como es bien sabido, la conformabilidad de un material depende de variables externamente controlables (deformación $(\varepsilon)$, velocidad de deformación $(\dot{\varepsilon})$ y temperatura $(\mathrm{T})$ de la pieza conformada) y de las características de fluencia inherentes al material. Hoy en día, el modelo dinámico de materiales (DMM) se ha convertido en un método muy prometedor para analizar y optimizar la conformabilidad de una amplia gama de materiales (Al Omar, 1996; Bakkali El Hassani et al., 2008; Al Omar et al., 2010; Alcelay et al., 2014; Jenab y Taheri, 2014; Alcelay, 2015; Shang et al., 2015). Este modelo fue desarrollado por primera vez en base a los principios fundamentales de la mecánica de medios continuos de la fluencia plástica en grandes deformaciones, usando los conceptos de modelización de los sistemas físicos y los principios de la termodinámica irreversible que describen la estabilidad y la auto-organización de los sistemas caóticos (Ziegler, 1963; Prigogine, 1978; Wellstead, 1979; Prasad et al. 1984; Prasad y Sasidhara, 1997; Prasad y Seshacharyulu, 1998). En este modelo, la pieza conformada se considera como un disipador de energía sin capacidad significativa para almacenarla. Además, su comportamiento de fluencia, impuesto por las variables del proceso de conformado (velocidad de deformación y temperatura), es no lineal y provoca cambios irreversibles en la microestructura que pueden ser beneficiosos (recristalización dinámica, deformación superplástica) o dañinos (fluencia localizada, bandas de cizalladura, cizalladura adiabática, formación de cavidades, rotura intercristalina, etc.). Desde el punto de vista industrial, la recristalización dinámica es el cambio microestructural más beneficioso que puede aparecer durante un proceso de conformado, permitiendo una mejora significativa de la conformabilidad intrínseca del material conformado mediante la reconstitución de la microestructura y afino de grano.

Para evaluar la conformabilidad de los materiales metálicos, durante procesos de conformado en caliente, Prasad (Prasad et al., 1984; Prasad y Sasidhara, 1997; Prasad y Seshacharyulu, 1998) ha propuesto construir mapas de procesado que permiten una caracterización explícita del comportamiento del material, bajo condiciones de conformado, en términos de cambios microestructurales. Estos mapas se componen de la superposición 
de dos tipos de mapas: un mapa de disipación de energía y un mapa de inestabilidad plástica, los dos desarrollados en base al DMM. El mapa de disipación de energía representa las características de disipación de energía a través de cambios microestructurales en términos de la eficiencia de disipación de energía expresada por el parámetro:

$\eta=\frac{2 m}{m+1}$

donde $m$ es el coeficiente de sensibilidad a la velocidad de deformación.

La variación del parámetro $\eta$, con la temperatura y con la velocidad de deformación, constituye el mapa de eficiencia de disipación de energía del material conformado. Este mapa ayuda a identificar las zonas óptimas de conformado y evitar las regiones consideradas de daño microestructural. Entre los zonas óptimas de conformado, el dominio asociado con la DRX es el más eficiente y se considera el más indicado para el conformado en caliente (Raj, 1981; Frost y Ashby, 1982; Prasad y Sasidhara, 1997). En consecuencia, estos mapas son de gran utilidad industrial porque permiten optimizar los parámetros de control de procesos industriales de conformado y producir piezas fiables y sin defectos (Prasad y Sasidhara, 1997).

Para la construcción de los mapas de inestabilidad plástica se evalúa el parámetro inestabilidad plástica $\xi(\dot{\varepsilon}, T)$ y se representa su variación en función de la temperatura y de la velocidad de deformación, a una deformación dada. El parámetro de inestabilidad plástica viene dado por la expresión siguiente:

$\xi(\dot{\varepsilon}, T)=\frac{\partial \log [\mathrm{m} / m+1]}{\partial \log \dot{\varepsilon}}+m$

Los mapas de inestabilidad plástica delimitan las zonas de daño microestructural. Estas zonas se caracterizan por valores negativos de $\xi(\dot{\varepsilon}, T)$ y son a evitar durante los procesos de conformado.

En la literatura son pocos los trabajos de investigación dedicados a la caracterización del comportamiento de fluencia de los aceros microaleados de medio carbono, durante un proceso de conformado en caliente, aplicando a la vez un modelo de RNA y el DMM (Alcelay et al., 2014; Alcelay, 2015). El presente trabajo pretende alcanzar dos objetivos principales; por una parte desarrollar un modelo de RNA para predecir el comportamiento de fluencia del acero estudiado, utilizando las curvas de fluencia obtenidas mediante ensayos de compresión en caliente realizados en el rango de temperaturas $900-1150{ }^{\circ} \mathrm{C}$ y velocidades de deformación $\dot{\varepsilon}=10^{-4}-10 \mathrm{~s}^{-1}$. Por otra parte, construir los mapas de procesado usando los valores de la tensión de fluencia predichos por el modelo de RNA.
Tabla 1. Composición química del acero estudiado ( $\%$ en peso)

\begin{tabular}{lcccccccc}
\hline $\mathbf{C}$ & $\mathbf{M n}$ & $\mathbf{S i}$ & $\mathbf{P}$ & $\mathbf{S}$ & $\mathbf{V}$ & $\mathbf{A l}$ & $\mathbf{T i}$ & $\mathbf{N p p m}$ \\
\hline 0,34 & 1,52 & 0,72 & 0,025 & 0,025 & 0,083 & 0,0145 & 0,018 & 0,0114 \\
\hline
\end{tabular}

\section{PROCEDIMIENTO EXPERIMENTAL}

El material utilizado en este estudio es un acero microaleado comercial de medio carbono cuya composición química se presenta en la Tabla 1. Para obtener las curvas de fluencia, se llevaron a cabo ensayos de compresión uniaxial sobre probetas cilíndricas de $11,4 \mathrm{~mm}$ de altura y 7,6 $\mathrm{mm}$ de radio, en un rango de temperaturas entre $900{ }^{\circ} \mathrm{C}$ y $1150{ }^{\circ} \mathrm{C}$ y velocidades de deformación entre $10^{-4}$ y $10 \mathrm{~s}^{-1}$. Para obtener la misma microestructura inicial en todos los ensayos, las probetas se precalentaban previamente durante 5 minutos a $1200{ }^{\circ} \mathrm{C}$ y luego se enfriaban hasta la temperatura de ensayo. Inmediatamente después de acabar el ensayo, las probetas eran templadas en agua y, posteriormente, preparadas para su estudio metalográfico.

\section{MODELO DE RNA PROPUESTO}

Una red neuronal es un modelo artificial altamente simplificado de la estructura del cerebro humano (Smith, 1993; Haykin, 1999; Hagan et al., 2002). Es un sistema para el tratamiento de información, cuya unidad básica de procesamiento se llama "neurona". En una RNA, las neuronas están distribuidas normalmente en capas conectadas entre sí: una capa de entrada, una o varias capas ocultas y una capa de salida. Las neuronas de la capa de entrada interactúan con el mundo real para recibir datos de entrada. Después de procesar estos datos, se envían a la capa oculta que a su vez los procesa y envía una respuesta a la capa de salida. La conexión entre las diferentes capas de neuronas está representada por lo que se denomina "fuerza de conexión" o "peso" (Zhao et al., 2014; Alcelay, 2015; Reddy et al., 2015). En general, un modelo de RNA se desarrolla en las siguientes etapas: (a) adquisición de datos experimentales; (b) especificación de los parámetros de entrada/salida; (c) análisis y procesamiento de los datos adquiridos; (d) entrenamiento de la RNA; (e) validación y test de la RNA entrenada y (f) evaluación del rendimiento del modelo de RNA.

En este estudio, para caracterizar el comportamiento de fluencia del acero microaleado, se ha propuesto un modelo de RNA de perceptrón multicapa con un algoritmo de aprendizaje de retropropagación del error. Este modelo se compone de: una capa de entrada, dos capas ocultas y una capa de salida (Fig. 1). La capa de entrada consiste en tres neuronas que representan los parámetros independientes 


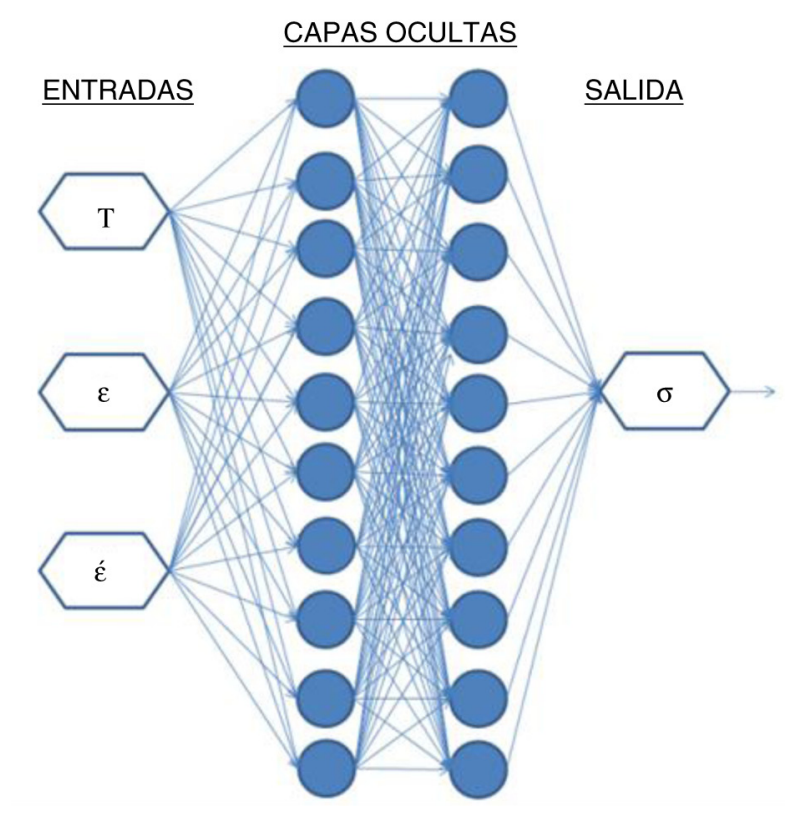

FIGURA 1. Arquitectura de la red neuronal.

del proceso de conformado: temperatura $(\mathrm{T})$, deformación $(\varepsilon)$ y velocidad de deformación $(\dot{\varepsilon})$. La capa de salida consiste en una sola neurona que representa la tensión de fluencia $\sigma$ del acero estudiado. $\mathrm{La}$ arquitectura de la red neuronal, así como su entrenamiento, aprendizaje y posterior validación se llevaron a cabo con el módulo de redes del programa Matlab. Para desarrollar el modelo de RNA se ha construido una base de datos (Tabla 2) que se compone de los valores experimentales obtenidos a partir de ensayos de compresión uniaxial en caliente a diferentes velocidades de deformación y temperaturas. Esta base de datos se ha utilizado para entrenar a la RNA y, también, para probar su fiabilidad con datos que no se han utilizado en el entrenamiento. Por tanto, para el entrenamiento se utilizaron 26 curvas de fluencia y para el test 16 de dichas curvas. Cabe mencionar que estos datos fueron elegidos aleatoriamente y que el número de datos de test concuerda con la regla general para verificar el correcto funcionamiento de la red (Smith, 1993; Haykin, 1999; Hagan et al., 2002).

La RNA requiere que tanto los datos de entrada como los de salida estén comprendidos entre 0 y 1 . Por lo tanto, los datos deben ser normalizados antes de introducirlos en la red. Para la normalización de $\mathrm{T}$ y de $\sigma$ se utiliza la siguiente ecuación:

$Z^{\prime}=\frac{Z-0,95 * Z_{\min }}{1,05^{*} Z_{\max }-0,95^{*} Z_{\min }}$

donde $\mathrm{Z}$ es el valor experimental ( $T$ y $\sigma$ ) y $Z^{\prime}$ es el valor normalizado de $Z$. Los valores máximos y mínimos de $Z^{\prime}$ se obtienen mediante $Z_{\max }$ y $Z_{\min }$, respectivamente. Sin embargo, la ecuación anterior no se puede utilizar para normalizar la velocidad de deformación $\dot{\varepsilon}$, ya que los valores normalizados de $\dot{\varepsilon}$ obtenidos son demasiado pequeños. Para evitar este problema, se utiliza una normalización logarítmica a través de la ecuación:

$$
\dot{\varepsilon}^{\prime}=\frac{5+\log \dot{\varepsilon}-0,95 *\left(5+\log \dot{\varepsilon}_{\min }\right)}{1,05 *\left(5+\log \dot{\varepsilon}_{\max }\right)-0,95 *\left(5+\log \dot{\varepsilon}_{\min }\right)}
$$

siendo $\dot{\varepsilon}$ el valor experimental, y $\dot{\varepsilon}^{\prime}$ el valor normalizado. La constante 5 se utiliza para que todos los valores normalizados sean positivos. En cuanto a la deformación, puesto que sus valores se encuentran entre 0 y 1 , no es necesario normalizarlos.

El entrenamiento del modelo desarrollado se llevó a cabo mediante el algoritmo Levenberg-Marquardt (LM); es uno de los algoritmos de aprendizaje más eficientes utilizados en el entrenamiento de las RNA (Smith, 1993; Haykin, 1999; Hagan et al., 2002; Alcelay, 2015). El ajuste de los pesos en el modelo se hace a través del algoritmo de retropropagación del error que permite minimizar el error cuadrático medio entre el valor de salida obtenido por el modelo de RNA y el valor real deseado, mediante el método del gradiente decreciente y una función de activación diferenciable. En el presente trabajo, la función sigmoidea ha sido utilizada como función de activación o de transferencia entre las capas ocultas. Esta función se expresa mediante:

TABLA 2. Base de datos utilizada para el desarrollo del modelo de RNA

\begin{tabular}{llll}
\hline$\dot{\varepsilon}\left(\mathbf{s}^{-\mathbf{1}}\right)$ & $\begin{array}{c}\text { Curvas de fluencia utilizadas para el } \\
\text { entrenamiento del modelo de RNA }\end{array}$ & \multicolumn{1}{c}{$\left(\mathbf{s}^{-\mathbf{1}}\right)$} & $\begin{array}{c}\text { Curvas de fluencia utilizadas para el } \\
\text { entrenamiento del modelo de RNA }\end{array}$ \\
\hline $10^{-4}$ & $900,1000,1050,1100\left({ }^{\circ} \mathrm{C}\right)$ & $3 \times 10^{-4}$ & $900,950,1000,1050\left({ }^{\circ} \mathrm{C}\right)$ \\
$10^{-3}$ & $900,950,1100,1150\left({ }^{\circ} \mathrm{C}\right)$ & $5 \times 10^{-3}$ & $900,1000\left({ }^{\circ} \mathrm{C}\right)$ \\
$10^{-2}$ & $900,950,1050,1100,1150\left({ }^{\circ} \mathrm{C}\right)$ & $3 \times 10^{-3}$ & $950,1050,1150\left({ }^{\circ} \mathrm{C}\right)$ \\
$10^{-1}$ & $900,950,1050,1100,1150\left({ }^{\circ} \mathrm{C}\right)$ & $5 \times 10^{-2}$ & $900,1000,1050\left({ }^{\circ} \mathrm{C}\right)$ \\
1 & $1000,1100\left({ }^{\circ} \mathrm{C}\right)$ & $3 \times 10^{-2}$ & $950,1050\left({ }^{\circ} \mathrm{C}\right)$ \\
3 & $1000,1050,1150\left({ }^{\circ} \mathrm{C}\right)$ & $10^{-1}$ & $1100{ }^{\circ} \mathrm{C}$ \\
10 & $950,1100,1150\left({ }^{\circ} \mathrm{C}\right)$ & 1 & $1050{ }^{\circ} \mathrm{C}$ \\
\hline
\end{tabular}




$$
f(x)=\frac{1}{1+e^{-x}}
$$

Después de varios ciclos de entrenamiento, se ha observado que se obtienen mejores resultados con una red que tiene dos capas ocultas de 10 neuronas cada una. Por lo tanto, la arquitectura de la ANN adoptada en este estudio es 3-10-10-1 (Fig. 1).

Para evaluar el rendimiento del modelo desarrollado, se han utilizado cuatro índices estadísticos estándar: el coeficiente de correlación $(R)$, el promedio de error absoluto relativo $(A A R E)$, la raíz cuadrada del error cuadrado medio $(R M S E)$ y el índice de dispersión (SI) (Zhao et al., 2014). Estos cuatro índices se definen a continuación:

$$
R=\frac{\sum_{i=1}^{N}\left(E_{i}-\bar{E}\right)\left(P_{i}-\bar{P}\right)}{\sqrt{\left(\sum_{i=1}^{N}\left(E_{i}-\bar{E}\right)^{2} \sum_{i=1}^{N}\left(P_{i}-\bar{P}\right)^{2}\right)}}
$$

$A A R E=\frac{1}{N} \sum_{i=1}^{N}\left[\frac{E_{i}-P_{i}}{E}\right] \times 100 \%$

$R M S E=\sqrt{\frac{1}{N} \sum_{i=1}^{N}\left(E_{i}-P_{i}\right)^{2}}$

$S I=\frac{R M S E}{\bar{E}}$

donde $E$ es el valor experimental y $P$ es el valor obtenido mediante el modelo de RNA; $\bar{E}$ y $\bar{P}$ son los valores medios de $E$ y $P$ respectivamente; $N$ es el número total de datos empleados en la investigación; $p$ corresponde al número de variables de salida.

Cabe mencionar que el algoritmo de aprendizaje BP se basa en un procedimiento iterativo para minimizar los índices $R M S E$ y $A A R E$; después de cada iteración, el valor del parámetro de salida se compara con el valor experimental, calculando los dos índices. El modelo se considera bueno cuando se logran valores muy bajos ( 0,0001). Pero, es importante tener en cuenta que valores muy altos del índice R (cerca de 1) no deberían siempre ser interpretados como evidencia de un buen rendimiento predictivo del modelo, porque estos valores no describen completamente la relación entre los valores experimentales y los valores teóricos del modelo (Ehrman et al., 2000; Phaniraj y Lahiri, 2003). Sin embargo, los cuatro índices estadísticos estándar tienen que ser utilizados juntos para una evaluación adecuada del rendimiento del modelo.

\section{RESULTADOS Y DISCUSIÓN}

\subsection{Predicción del comportamiento de fluencia usando el modelo de RNA}

La Tabla 3 recoge los valores estadísticos estándar obtenidos mediante el entrenamiento y test del modelo de RNA desarrollado en este trabajo. Según se observa en esta tabla, existe una excelente concordancia entre los datos experimentales y los valores teóricos del modelo; los porcentajes de error respecto a los experimentales son inferiores al 5\%, tanto para el RMSE, AARE y SI. Además, el coeficiente de correlación lineal $R$ es una buena medida de la bondad del ajuste lineal entre las variables experimentales y teóricas. A la vista de los valores obtenidos para este coeficiente (muy cercanos a 1) se puede afirmar que el ajuste lineal es perfecto.

La Fig. 2 muestra la regresión entre los valores experimentales y los logrados mediante el modelo de RNA. En esta figura se dibuja una línea inclinada a $45^{\circ}$ respecto a la horizontal. Para un ajuste perfecto, todos los puntos deben estar sobre esta línea y tal como se puede observar en la figura, los valores obtenidos mediante el modelo de red están en muy buena concordancia con los valores obtenidos experimentalmente; la mayor parte de los puntos se encuentran sobre la línea de $45^{\circ}$. Los coeficientes de correlación obtenidos para el entrenamiento y test de la red son muy cercanos a 1: 0,9993 y 0,9975, respectivamente. Estos resultados indican que las predicciones del modelo de RNA son muy precisas y la red ha sido entrenada satisfactoriamente y podría ser aplicada para predecir el comportamiento de fluencia del acero estudiado.

Además, y para garantizar la consistencia y la fiabilidad del rendimiento del modelo de RNA, se ha llevado a cabo un análisis estadístico del error relativo. El error entre los datos experimentales y los datos teóricos del modelo de red se puede calcular a través de la siguiente expresión:

Error relativo $=\left(\frac{E_{i}-P_{i}}{E}\right) \times 100 \%$

La distribución del error de predicción del modelo de RNA para el entrenamiento, prueba y test se muestra en la Fig. 3. Esta figura indica que la distribución del error relativo de los dos conjuntos de datos (entrenamiento y test) sigue una distribución típica de Gauss y, además, para más del

TABLA 3. Rendimiento del modelo RNA para el entrenamiento y test de los datos del acero estudiado

\begin{tabular}{lcccc}
\hline$\dot{\boldsymbol{\varepsilon}}\left(\mathbf{s}^{\mathbf{- 1}}\right)$ & $\mathbf{R}$ & RMSE (\%) & AARE (\%) & SI \\
\hline Entrenamiento & 0,9993 & 1,4579 & 1,4579 & 0,0203 \\
Test & 0,9975 & 4,3665 & 3,5850 & 0,0601 \\
\hline
\end{tabular}



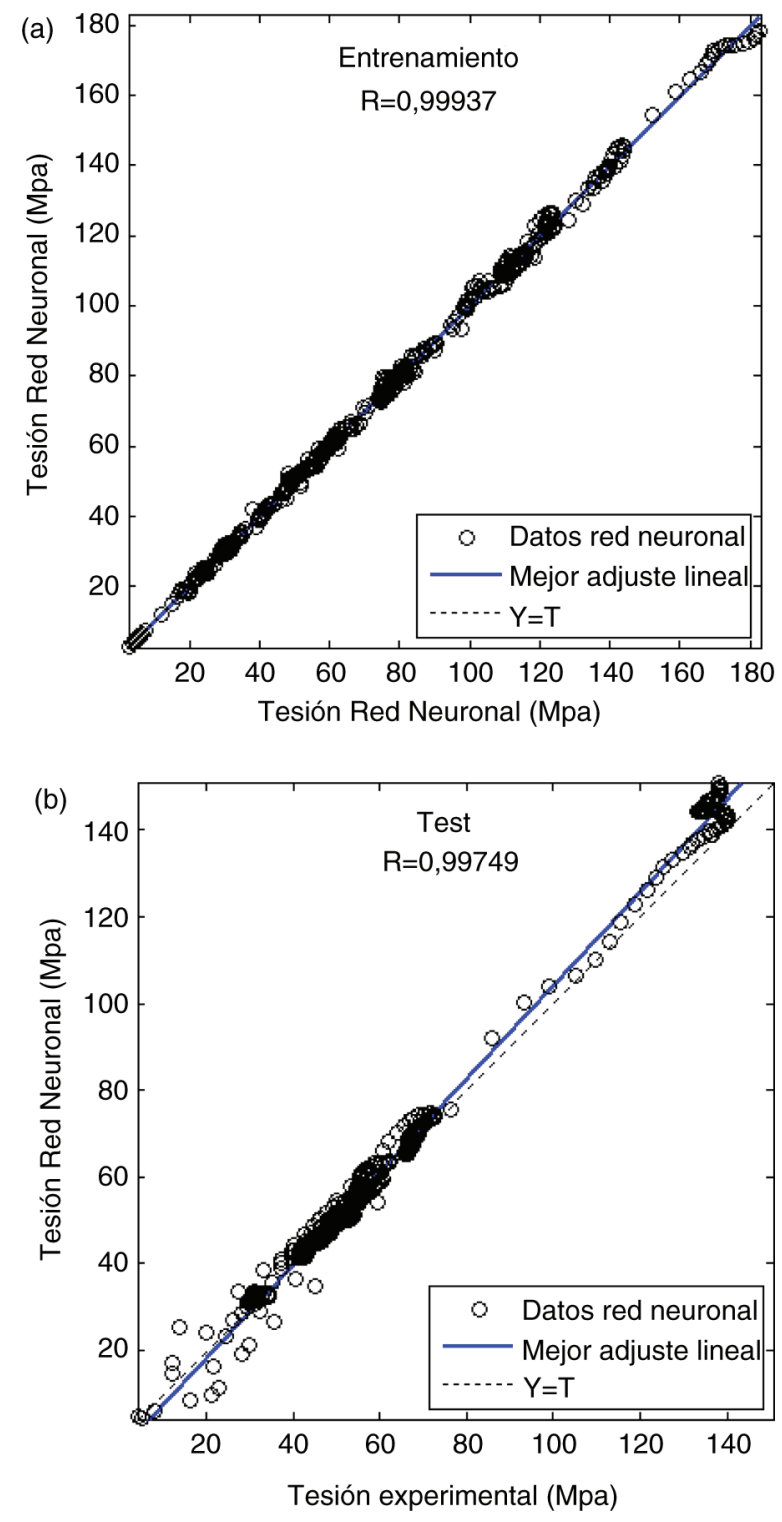

FIgURA 2. Comparación entre resultados experimentales y resultados obtenidos por el modelo de RNA para: (a) entrenamiento y (b) test.

95\% de los datos del entrenamiento, el error relativo se encuentra dentro del $\pm 10 \%$. Para los datos del test, el error relativo está dentro de $\pm 5 \%$ para más del $88 \%$ de datos. En consecuencia, se confirma el buen rendimiento del modelo de RNA propuesto. Es importante señalar que el error de predicción no puede atribuirse solamente a la capacidad de predicción del modelo, sino en parte al ruido experimental que aparece durante la realización de los ensayos de compresión en caliente.

En la Fig. 4 se muestra una comparación entre las curvas de fluencia experimentales con las obtenidas con el modelo de RNA desarrollado, a diferentes velocidades de deformación y temperaturas. Puede observarse que en general existe una muy
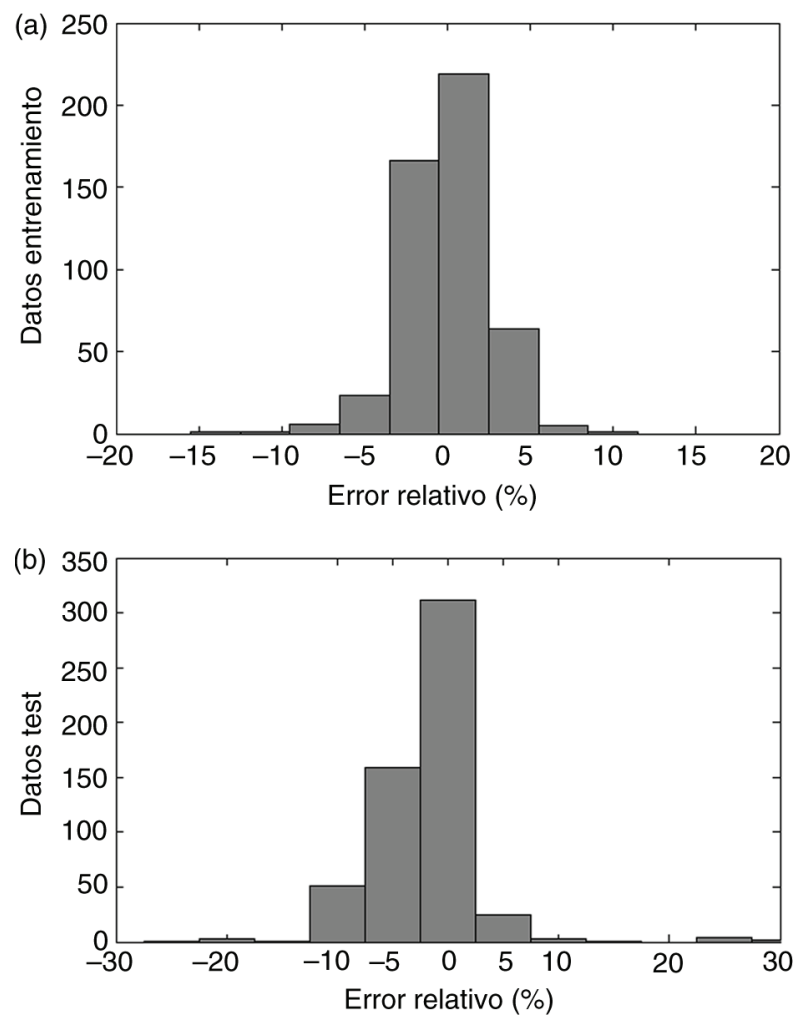

FIgURA 3. Análisis estadístico del error de predicción del modelo de RNA para: (a) entrenamiento y (b) test de datos.

buena concordancia entre los resultados del modelo de RNA y los de los ensayos experimentales, bajo diferentes condiciones de deformación. El modelo desarrollado predice satisfactoriamente, en las curvas de fluencia, la tendencia al endurecimiento por deformación y al ablandamiento dinámico posterior. Sin embargo, a pesar de esta excelente concordancia, se debe mencionar que el modelo de RNA no es capaz de modelizar el comportamiento cíclico de la recristalización dinámica, a bajas velocidades de deformación y altas temperaturas. Esto se debe a que el modelo de RNA utiliza el método del gradiente descendiente para minimizar la función de error en el espacio de pesos.

\subsection{Construcción de los mapas de procesado usando el modelo de RNA}

Para comprobar la predictibilidad del modelo de RNA propuesto en este trabajo, se han construido los mapas de procesado en base al DMM y usando los valores de la tensión de fluencia predichos por el modelo de RNA.

\subsubsection{Mapa de eficiencia de disipación de energía}

La Fig. 5 muestra el mapa de contornos de iso-eficiencia de disipación de energía del acero estudiado obtenido a deformación de 0,6 (que es, 
(a)

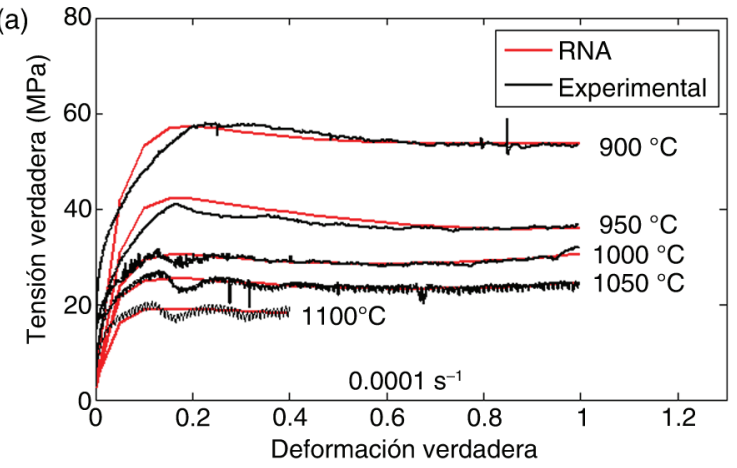

(c)

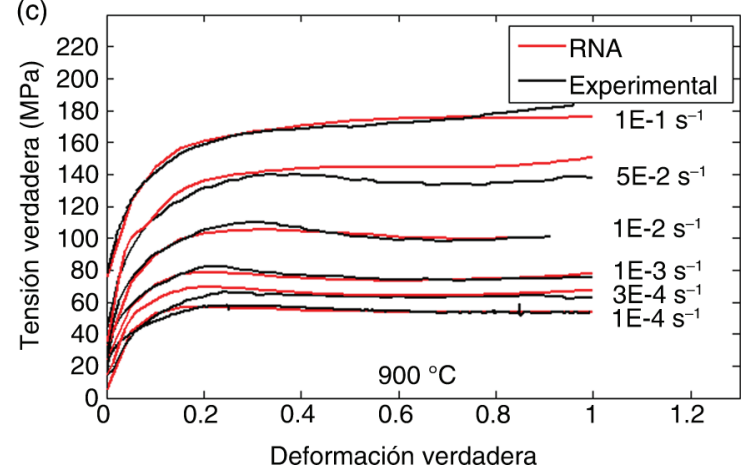

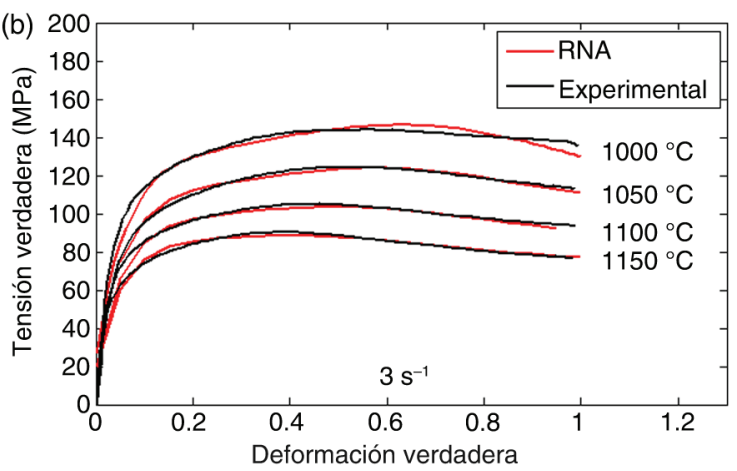

(d)

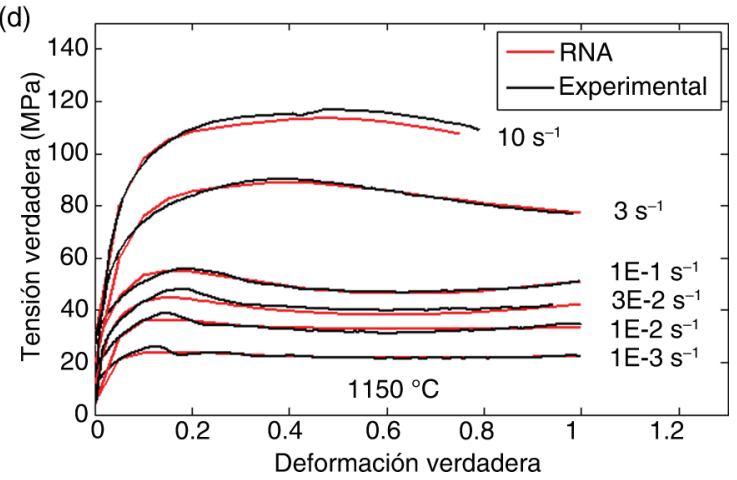

FIGURA 4. Comparación de las curvas de fluencia experimentales y teóricas del acero estudiado a varias T y $\dot{\varepsilon}$.

aproximadamente, la deformación correspondiente a fluencia de estado estable). Tal como se ha mencionado arriba, la eficiencia de disipación de energía ( $)$ se obtiene aplicando la Ec. (1). Caber recordar que la eficiencia de disipación de energía caracteriza la microestructura disipativa del material, conformado bajo diferentes condiciones de temperatura y velocidad de deformación, y la interpretación del mapa de iso-eficiencia se basa en el hecho de

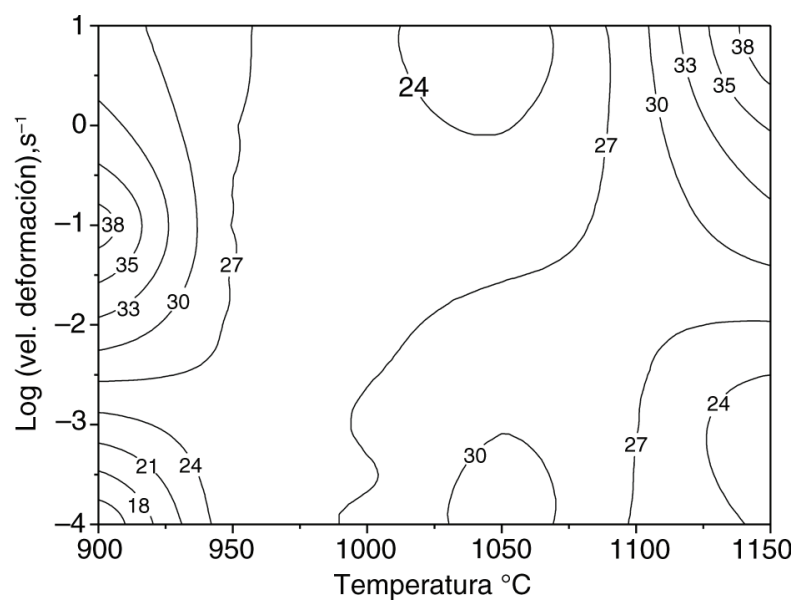

Figura 5. Mapa de eficiencia de disipación de energía obtenido mediante el modelo de RNA para la deformación $\varepsilon=0,6$. que algunas de las manifestaciones de inestabilidad plástica se caracterizan por dominios con mínimos o valores muy altos del parámetro $\eta$.

La observación de la Fig. 5 revela la existencia de dos dominios caracterizados por un máximo de eficiencia. El primero, aparece centrado a $1150{ }^{\circ} \mathrm{C}$ y $10 \mathrm{~s}^{-1}$ con una eficiencia máxima de aproximadamente 38\%; es el dominio de la DRX (Al Omar, 1996). Esta correlación se confirma mediante las curvas de fluencia obtenidas bajo diferentes combinaciones de temperatura y velocidad de deformación en este dominio y muestran claramente un ablandamiento continuo con un comportamiento de pico simple. El segundo dominio se produce en la región de bajas temperaturas y velocidades de deformación moderadas (centrado a aproximadamente $900{ }^{\circ} \mathrm{C} \mathrm{y} 0,1 \mathrm{~s}^{-1}$ ). En este caso de bajas temperaturas y velocidades de deformación intermedias es de esperar que actúe la DRV; las curvas típicas de fluencia dentro de este dominio son características de la DRV. En un proceso de conformado en caliente, tanto la DRX como la DRV se consideran mecanismos beneficiosos para la microestructura y, en consecuencia, las propiedades mecánicas del material conformado, ya que proporcionan una fluencia estable y mejora la conformabilidad del material (Al Omar, 1996; Prasad y Sasidhara, 1997). Por tanto, puede decirse que estos dominios son los dominios más seguros para el conformado del acero estudiado. 


\subsubsection{Mapas de inestabilidad plástica}

Usando los valores del modelo de RNA se representa en la Fig. 6 la variación del parámetro de inestabilidad plástica $\xi$ (Ec. (2)) con la velocidad de deformación y la temperatura para la deformación de $\varepsilon=0,6$. Como se ha mencionado anteriormente, cuanto mayor es la magnitud negativa del parámetro $\xi$, mayor es la posibilidad de aparición de alguna manifestación de inestabilidad plástica (Prasad y Sasidhara, 1997). Así, con el fin de conformar siempre en condiciones de fluencia estable, los dominios de posibles inestabilidades deben ser evitados durante los procesos de conformado en caliente.

El mapa mostrado en la Fig. 6 no revela ningún tipo de inestabilidad plástica y está en total acuerdo con el mapa de iso-eficiencia de disipación de energía. Como es bien sabido, los dominios estables correspondientes a DRX y DRV se caracterizan por altos valores del parámetro $\xi$ y cuanto más grande es el valor positivo de $\xi$ mayor es la estabilidad del comportamiento de fluencia del acero conformado (Al Omar, 1996; Prasad y Sasidhara, 1997). Este mapa exhibe un dominio centrado en $1150{ }^{\circ} \mathrm{C}$ y $10 \mathrm{~s}^{-1}$ con un máximo de $\xi$ de aproximadamente 0,24 . Este dominio puede atribuirse fácilmente a la DRX de pico simple, observado en el mapa de la Fig. 5 y situado en la misma posición. El dominio de la DRV se ha detectado en la zona extendida de moderadas hacia altas $\dot{\varepsilon}$ y $T=900{ }^{\circ} \mathrm{C}$, con una valor de $\xi=0,30$. Este dominio coincide con el dominio de la DRV identificado en el mapa de la Fig. 6 con una pequeña diferencia en la posición.

Los mapas de las Figs. 5 y 6 ponen de manifiesto el buen acuerdo que existe entre los resultados experimentales (Al Omar, 1996; Al Omar et al., 1996; Al Omar et al., 1997) y los del modelo de RNA, ya que tanto el mapa de iso-eficiencia de disipación de energía como el de inestabilidad plástica muestran la misma

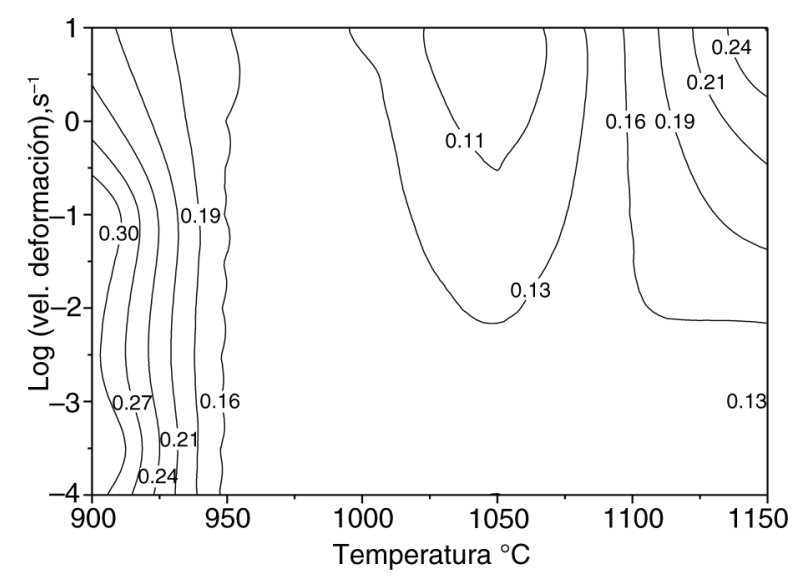

Figura 6. Mapa de inestabilidad obtenido mediante el modelo de RNA para $\varepsilon=0,6$ tendencia que los mapas construidos utilizando valores experimentales de la tensión de fluencia.

\section{CONCLUSIONES}

En este trabajo, se ha utilizado con éxito una red neuronal artificial para caracterizar el comportamiento termo-mecánico de un acero microlaeado de medio carbono durante un proceso de conformado en caliente. El modelo de RNA desarrollado consiste en una red de perceptrón multicapa con un algoritmo de aprendizaje de retropropagación. Esta red está compuesta por una capa de entrada de tres neuronas (deformación, velocidad de deformación y temperatura), dos capas ocultas con 10 neuronas cada una y una capa de salida con una neurona (tensión de fluencia). De esta manera se ha podido modelizar la curva de fluencia del acero estudiado como una función implícita de la deformación, velocidad de deformación y temperatura. Las curvas de fluencia, obtenidas mediante los ensayos de compresión en caliente, han sido utilizadas para entrenar el modelo de RNA propuesto. Los resultados obtenidos indican una buena correlación entre los valores experimentales y los valores teóricos del modelo de RNA. El análisis de los índices estadísticos estándar, utilizados en este trabajo, ha puesto de manifiesto la excelente capacidad de predicción del modelo. Para más del 95\% de los datos del entrenamiento, el error relativo se encuentra dentro del $\pm 10 \%$, mientras que para los datos del test, el error relativo está dentro de $\pm 5 \%$ para más del $88 \%$ de datos. Por lo tanto, el modelo de RNA puede considerarse como una herramienta eficiente para caracterizar de manera rápida y precisa el comportamiento de fluencia de los aceros microaleados de medio de carbono, sin necesidad de encontrar un gran número de constantes de material, relacionadas con el método de regresión empleado para determinar la ecuación constitutiva. Además, se ha podido comprobar que la metodología utilizada es simple y general y es adecuada para cualquier material.

Por último, con el fin de comprobar la fiabilidad del modelo de RNA propuesto, se han construido, para la deformación $\varepsilon=0,6$, los mapas de procesado, basados en el DMM, utilizando valores de la tensión de fluencia predichos por el modelo. Estos mapas son prácticamente similares a los construidos utilizando valores experimentales de tensión de fluencia. Asimismo estos mapas revelan la existencia de

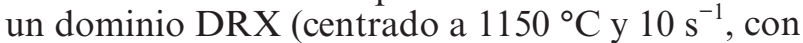
un pico de eficiencia del $38 \%$ ) que puede ser considerado como el dominio más seguro para el conformado en caliente del acero estudiado. Además, muestran un dominio DRV a bajas temperaturas y moderada velocidad de deformación.

Los resultados obtenidos en este trabajo sugieren, para futuros trabajos de investigación, la implementación del modelo de RNA en un código comercial 
de cálculo por elementos finitos para simular de manera más satisfactoria el comportamiento de fluencia de los aceros microaleados.

\section{AGRADECIMIENTOS}

Los autores desean agradecer a la CICYT (España) la financiación económica a través del Proyecto DPI2013-40882-P.

\section{REFERENCIAS}

Akbari, Z., Mirzadeh, H., Cabrera, J.M. (2015). A simple constitutive model for predicting flow stress of medium carbon microalloyed steel during hot deformation. Mater. Design 77, 126-131. http://dx.doi.org/10.1016/j.matdes. 2015.04.005.

Al Omar, A. (1996). Caracterización dinámica de dos aceros microaleados de medio carbono mediante ensayos de compresión a alta temperatura. Aplicación de mapas de procesado, Tesis Doctoral, Univ. Politécnica de Cataluña, Barcelona.

Al Omar, A., Cabrera, J.M., Prado, J.M. (1996). Characterization of the hot deformation in a microalloyed medium carbon steel using processing maps. Scripta Mater. 34(8), 1303-1308. http://dx.doi.org/10.1016/1359-6462(95)00651-6.

Al Omar, A., Cabrera, J.M., Prado, J.M. (1997), Aplicación de los mapas de procesado en la optimización de los parámetros de un proceso de conformado en caliente. II parte. Mapas de procesado de un acero microaleado con un contenido medio de carbono. Rev. Metal. 33 (3), 153-160. http://dx.doi.org/10.3989/revmetalm.1997.v33.i3.858

Al Omar, A., Alcelay, I., Prado, J.M. (2010). Criterios de predicción de inestabilidades plásticas en procesos de conformado en caliente. (Parte II. Caracterización de la fluencia plástica de un acero microaleado de medio carbono usando criterios continuos y fenomenológicos). Rev. Metal. 46 (2), 162-172. http://dx.doi.org/10.3989/revmetalm.0937.

Alcelay, I., Al Omar, A., Prado, J.M. (2014). Characterization of hot flow behaviour and deformation stability of microalloyed steel using artificial neural networks and dynamic material model. Int. J. Mater. Res. 105 (8), 743-754. http:// dx.doi.org/10.3139/146.111077.

Alcelay, I. (2015). Modelización de los procesos de conformado en caliente de aceros microaleados de medio carbono mediante la aplicación de redes neuronales artificiales, Tesis Doctoral, Univ. Politécnica de Cataluña, Barcelona.

Bakkali-El Hassani, F., Chenaoui, A., Dkiouak, R., Elbakkali, L., Al Omar, A. (2008). Characterization of deformation stability of medium carbon microalloyed steel during hot forging using phenomenological and continuum criteria. J. Mater. Process. Tech. 199 (1-3), 140-149. http://dx.doi. org/10.1016/j.jmatprotec.2007.08.004

Cabrera, J.M., Al Omar, A., Prado, J.M., Jonas, J.J. (1997). Modeling the flow behavior of a medium carbon microalloyed steel under hot working conditions. Metall. Mater. Trans. A 28 (11), 2233-2244. http://dx.doi.org/10.1007/ s11661-997-0181-8.

Ceschini, L., Marconi, A., Martini, C., Morri, A., Di Schino, A. (2013). Tensile and impact behaviour of a microalloyed medium carbon steel: Effect of the cooling condition and corresponding microstructure. Mater. Design 45, 171-178. http://dx.doi.org/10.1016/j.matdes.2012.08.063

Chai, R.X., Guo, C., Yu, L. (2012). Two flowing stress models for hot deformation of XC45 steel at high temperature. Mat. Sci. Eng. A-Struct. 534, 101-110. http://dx.doi.org/10.1016/j. msea.2011.11.047.

Da Silva, M.L.N., Regone, W., Button, S.T. (2006). Microstructure and mechanical properties of microalloyed steel forgings manufactured from cross-wedge-rolled preforms. Scripta Mater. 54 (2), 213-217. http://dx.doi.org/10.1016/j. scriptamat.2005.09.023.
Ehrman, J.M., Higuchi, K., Clair, T.A. (2000). Backcasting to test the use of neural networks for predicting runoff in canadian rivers. Can. Water Resour. J. 25 (3), 279-291. http://dx.doi.org/10.4296/cwrj2503279.

Frost, H.J., Ashby, M.F. (1982). Deformation-mechanism Maps: The Plasticity and Creep of Metals and Ceramics, Chapter: 1-5, Ed. Pergamon Press, Oxford, England.

Gupta, A.K., Singh, S.K., Reddy, S., Hariharan, G. (2012). Prediction of flow stress in dynamic strain aging regime of austenitic stainless steel 316 using artificial neural network. Mater. Design 35, 589-595. http://dx.doi.org/10.1016/j. matdes.2011.09.060.

Hagan, M.T., Demuth, H.B., Beale, M. (2002). Neural Network Design, Thomson Learning, Singapur.

Haykin, S. (1999). Neural networks: A comprehensive foundation, Prentice Hall International, Englewood Cliffs, USA.

Hui, W., Zhang, Y., Shao, C., Chen, S., Zhao, X., Dong, H. (2015). Microstructural effects on high-cycle fatigue properties of microalloyed medium carbon steel 38MnVS. Mat. Sci. Eng. A-Struct. 640, 147-153. http://dx.doi.org/10.1016/j. msea.2015.05.054

Jahazi, M., Eghbali, B. (2001). The influence of hot forging conditions on the microstructure and mechanical properties of two microalloyed steels. J. Mater. Process. Tech. 113 (1-3), 594-598. http://dx.doi.org/10.1016/S0924-0136(01) 00599-4.

Jenab, A., Taheri, A.K. (2014). Experimental investigation of the hot deformation behavior of AA7075: Development and comparison of flow localization parameter and dynamic material model processing maps. Int. J. Mech. Sci. 78, 97-105. http://dx.doi.org/10.1016/j.ijmecsci.2013. 11.003 .

Kaynar, A., Gündüz, S., Türkmen, M. (2013). Investigation on the behaviour of medium carbon and vanadium microalloyed steels by hot forging test. Mater. Design 51, 819-825. http://dx.doi.org/10.1016/j.matdes.2013.04.102

Naylor, D.J. (1998). Microalloyed forging steels. Mater. Sci. Forum 284-286, 83-94. http://dx.doi.org/10.4028/www. scientific.net/MSF.284-286.83

Phaniraj, M.P., Lahiri, A.K. (2003). The applicability of neural network model to predict flow stress for carbon steels. J. Mater. Process. Tech. 141 (2), 219-227. http://dx.doi. org/10.1016/S0924-0136(02)01123-8.

Prasad, Y.V.R.K., Gegel, H.L., Doraivelu, S.M., Malas, J.C., Morgan, J.T., Lark, K.A., Barker, D.R. (1984). Modeling of dynamic material behavior in hot deformation: Forging of Ti-6242. Metall. Trans. A 15 (10), 1883-1892. http:// dx.doi.org/10.1007/BF02664902.

Prasad, Y.V.R.K., Sasidhara S. (1997). Hot Working Guide: A Compendium of Processing Maps, ASM International, Materials Park, Ohio, USA.

Prasad, Y.V.R.K., Seshacharyulu, T. (1998). Modelling of hot deformation for microstructural control. Int. Mater. Rev. 43 (6), 243-258. http://dx.doi.org/10.1179/095066098790105618.

Prigogine, I. (1978). Time, Structure and Fluctuations. Science 201 (4358), 777-785. http://dx.doi.org/10.1126/science.201. 4358.777.

Raj, R. (1981). Development of a processing map for use in warm-forming and hot-forming processes. Metall. Trans. A 12 (6), 1089-1097. http://dx.doi.org/10.1007/ BF02643490.

Reddy, N.S., Panigrahi, B.B., Ho, C.M., Kim, J.H., Lee, C.S. (2015). Artificial neural network modeling on the relative importance of alloying elements and heat treatment temperature to the stability of $\alpha$ and $\beta$ phase in titanium alloys. Comp. Mater. Sci. 107, 175-183. http://dx.doi.org/10.1016/j. commatsci.2015.05.026.

Shaban, M., Eghbali, B. (2010). Determination of critical conditions for dynamic recrystallization of a microalloyed steel. Mater. Sci. Eng. A 527 (16-17), 4320-4325. http://dx.doi. org/10.1016/j.msea.2010.03.086.

Shang, X., Zhou, J., Wang, X., Luo, Y. (2015). Optimizing and identifying the process parameters of AZ31 magnesium alloy in hot compression on the base of processing maps. J. Alloy. Compd. 629, 155-161. http://dx.doi.org/10.1016/j. jallcom.2014.12.251. 
Smith, M. (1993). Neural networks for statistical modelling, Van Nostrand Reinhold, Nueva York, USA.

Wellstead, P.E. (1979). Introduction to Pysical Systems Modelling, Ed. Academic Press, London, England.

Zhao, J., Ding, H., Zhao, W., Huang, M., Wei, D., Jiang, Z (2014). Modelling of the hot deformation behaviour of a titanium alloy using constitutive equations and artificial neural network. Comp. Mater. Sci. 92, 47-56. http://dx.doi. org/10.1016/j.commatsci.2014.05.040.

Ziegler, H. (1963). Progress in solid mechanica, Vol. 4, Ed. I.N. Sneddon y R. Hill, John Wiley \& Sons, Nueva York, USA. 